\title{
An in situ impedance measurement set-up for high sound pressure levels
}

\author{
A.Grosso ${ }^{1}$, E.Tijs ${ }^{1}$, B.Zajamsek ${ }^{1}$ \\ ${ }^{1}$ Microflown Technologies, Tivolilaan 205, Arnhem, Netherlands, \\ grosso@microflown.com \\ tijs@microflown.com \\ zajamsek@microflown.com
}

\begin{abstract}
In aerospace and related industries there is a request for acoustic impedance measurements under high sound pressure levels. Standard measurement techniques do not satisfy testing requirements or are sometime not applicable. In this paper a newly developed measurement system is introduced which can be used to measure materials properties under high sound pressure levels. The acoustic impedance can be measured in situ in a wide frequency range, from $100 \mathrm{~Hz}$ up to $8 \mathrm{kHz}$. From this test the material absorption and reflection can be calculated. The method imposes only few restrictions on the specimen in terms of shape or mounting conditions. The system has been successfully tested on a highly reactive aircraft engine linear sample; the results are presented in this paper.
\end{abstract}

Key words: Impedance, in situ, absorption, aerospace, reflection

\section{Introduction}

Acoustic properties of materials are measured traditionally by standardized methods, such as reverberant room, alpha cabin or in the kund's tube. Although those methods are widely used there are limitations in term of applicability and cost.

Since many years, an alternative measurement technique is available, that utilizes a PU probe, with which the sound pressure and particle velocity can be measured. This technique enables to test materials properties in situ, without modifying the structure.

In this paper three types of measurements are discussed. Firstly, measurements are analyzed which are performed on a jet engine intake liner with a standard impedance setup and, for comparison and to study flange size effects, with a Kundt's tube.

In the second measurement the same liner is measured with a scanning technique called "Scan \& Paint". With this technique very high spatial resolution is achieved to show local variation of the impedance of the sample under test.

In the third measurement a new impedance setup that generates sound pressure levels of about $150 \mathrm{~dB}$ is tested on the liner. The results are compared to those of the standard PU method.

\section{Jet engine intake liners}

Perforated liner samples are frequently used to attenuate the acoustic emission of aircraft jet engines. These liners are made from metals instead of fabric or plastics because large temperature variations and high mechanical stresses are experienced during flights. They usually consists of (a sandwich of) honeycomb structure(s) with a perforated top layer that is a light, but strong (see e.g. Fig.1).

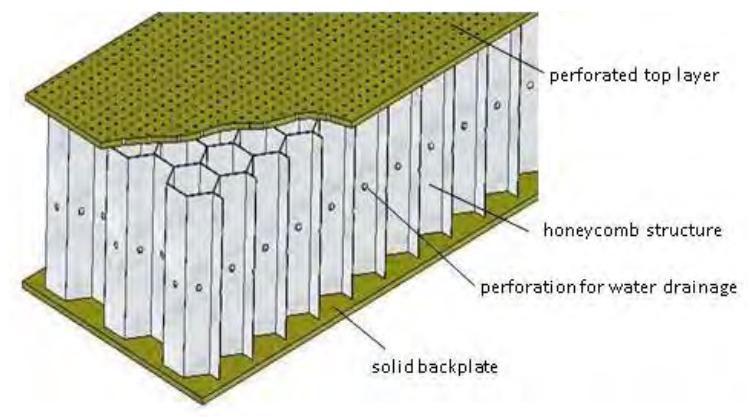

Fig. 1: Perforated liner for aerospace application.

In order to determine their acoustic performance various methods are used; e.g. open Kundt's tubes, simulations, and insertion loss measurements. The open Kundt's tube is most frequently used. In situ measurement 
methods are usually not applied because of the small size of such samples. In this paper results of various measurements on liners with are presented and investigated.

\section{PU in situ impedance method}

With the impedance setup the acoustic impedance, reflection, and absorption can be measured close to material surface. It involves a PU probe that is positioned close to the material and a sound source positioned at a certain distance that generates noise (Fig.2). The impedance can be estimated by the complex ratio of pressure and particle velocity [5]. Several theoretical models can be applied to correct for the spherical sound field that is present, and estimate the surface impedance, the reflection coefficient and the absorption coefficient, see e.g. [4].

Although the probe is able to measure in the whole audible range, to the low frequency limit is about 100 to $300 \mathrm{~Hz}$ mostly because of the low sound pressure emission from the loudspeaker and because of limitations of the absorption models.

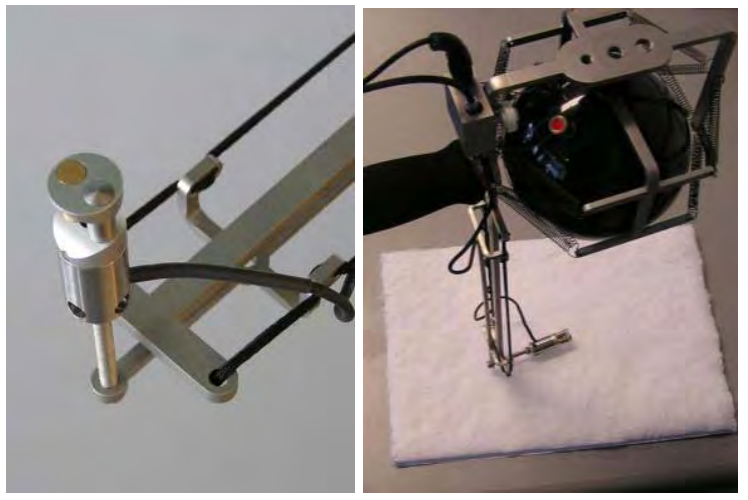

Fig. 2: Left: $P U$ probe, right: Hand-held $P U$ impedance set-up.

The measurement procedure involves two steps which each take approx. 10 s; i.e. firstly a calibration measurement without sample is performed, secondly a measurement is performed with the sample. Both measurements can be performed in a regular room such as office or in highly reverberant environment such as car interior. Undesirable reflections are removed via time-windowing by the impulse response technique or by a moving average in the frequency domain [2, 3]. It has been demonstrated that the results from such a calibration test are similar to tests performed in an anechoic calibration. The usability of above mentioned techniques depends on the environment and measurement conditions.

\section{Kundt's tube comparison - flange size effect}

In [7] a liner sample that comprises perforated honeycomb cells was measured with an open Kundt's tube and the PU technique. The cavities of this liner were connected to allow water to drain toward the bottom of the engine. With the method a small surface area was measured, thus the effective 'absorption' of it could be calculated [5].

Interesting observations were made when comparing the results of both methods. The results from the in situ method were similar to those obtained from the sample without perforated honeycomb cells. However, this was not the case for the Kundt's tube. To investigate the matter further, a special Kundt's tube was made onto which flanges with different sizes could be mounted. Three measurements were performed with this Kundt's tube: i.e. one without a flange, one with a flange of $115 \mathrm{~mm} \times 115 \mathrm{~mm}$ (the standard size), and one with a large flange that covered a large part of the sample. With the PU method an additional measurement with a flange was performed in order to investigate the effect. The results of these measurements are shown in Error! Reference source not found.3.

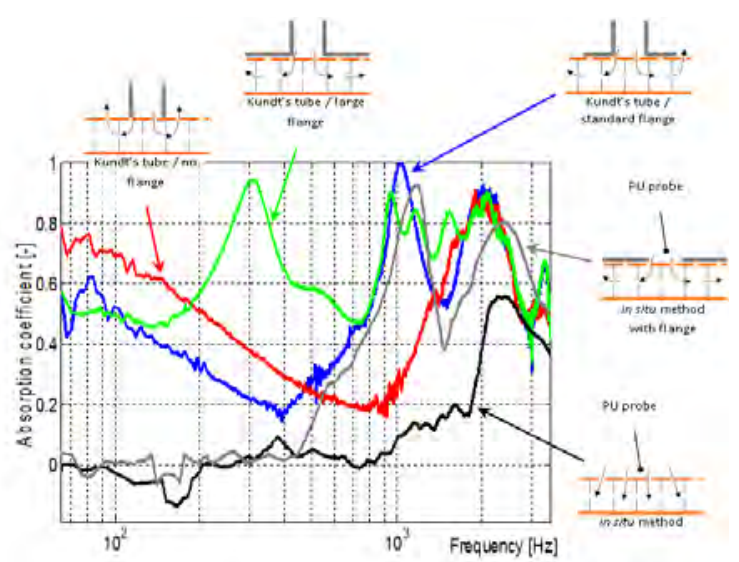

Fig. 3: Variation of flange size. Jet engine liner 'absorption' measured in situ and with the Kundt's tube.

The 'absorption' that was measured with the PU method (black line) is not only lower than the absorption measured by the Kundt's tube with standard flange (blue line), but even first peak around $1 \mathrm{kHz}$ that appears with the Kundt's tube is not found. The second peak is also different and is positioned at $2.5 \mathrm{kHz}$ instead of $2 \mathrm{kHz}$. It is unlikely that these discrepancies are caused by the type of sensors that are used because the principles of measuring the impedance in a Kundt's tube and in situ are similar. Also, the absorption models that are used are similar in both cases, except that the PU method involves a correction for spherical waves. 
In order to find the cause of these discrepancies the results of the Kundt's tube with different flange sizes are analyzed. The outcome of the Kundt's tube is questionable, as it seems to vary depending on the size of the flange. On one hand the flange size needs to be large to prevent sound leakage that occurs because all cavities are connected. The absorption is overestimated due to such a leakage, mainly at low frequencies. A value of 0.7 is measured at $100 \mathrm{~Hz}$ in this case, which is unrealistic for such thin samples. On the other hand the flange size should be small in order to prevent that additional standing waves occur inside the sample. The $1 \mathrm{kHz}$ peak is not measured without flange (red line). Additional modes are measured for larger flanges (see e.g. the green line).

Next, the in situ measurement with flange is examined (black vs. grey line). Higher 'absorption' values are measured, which are similar to those obtained with the Kundt's tube with standard flange (grey vs. blue line, $>400 \mathrm{~Hz}$ ). It can therefore be concluded that measurement errors are made when a flange is used.

The tests revealed that more accurate results can be obtained with PU mothod for liners with interconnected honeycomb cells.

\section{Measurement with Scan \& Paint system}

In situ impedance measurements are usually performed at one spot, so the local absorption properties can be estimated and visualized in the frequency domain. A comparison of different measurement points is possible by using a "point by point" technique, but usually such measurements are time-consuming. A new scanning measurement technique, called Scan \& Paint, offers the possibility to visualize the absorption properties of a sample with very higher spatial resolution and shorter measurement time. This method comprises a simultaneous measurement of the acoustic signals and a video camera that captures the movement of the acoustic measurement system as it is scanned over the surface. Here, the acoustic signals are acquired with the PU absorption setup as is shown in fig 1 . The position of the probe at each particular time of the record is determined by tracking a color mark on the probe in the video. Thereafter, the measurement positions are overlaid on a static background image. The sound pressure and the particle velocity, and ultimately the absorption, are calculated from a short section of the time signals of the sensors that starts just before and ends just after the time each probe position detection. With this technique is possible to quickly visualize the overall absorption properties of large surfaces, which allows identification of regions with high absorption coefficients and in-homogeneities of materials.

In the following measurement this technique is used to scan a small portion of a liner to demonstrate the spatial resolution of the method. In order to increase the spatial resolution a miniature combined pressurevelocity probe called PU-match probe (Fig.4) is used. This probe can be positioned very close to the surface because of its small size $(2 \mathrm{~mm}$ in this case).

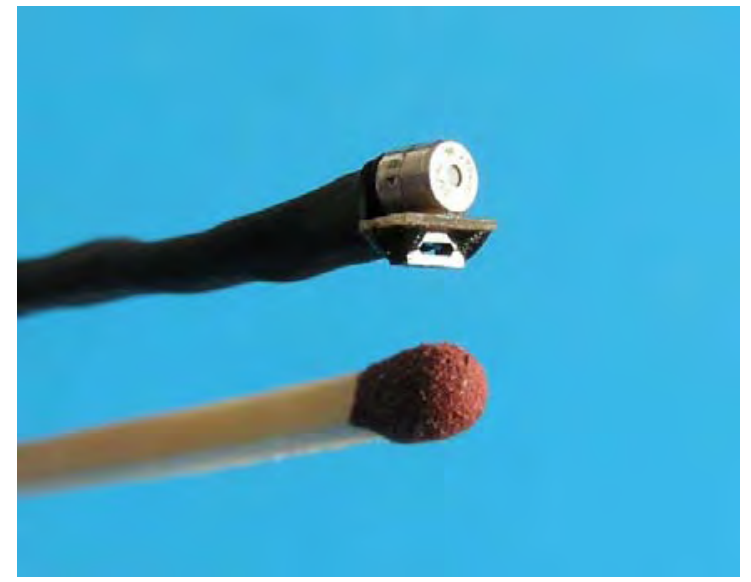

Fig. 4: PU match probe

To obtain a good accuracy is necessary to keep a constant distance probe-sample; for this reason the video camera and the liner are both positioned on a metal plate which is moved, while the measurement set-up is fixed on a tripod and does not move. The video frame rate is fixed to 20fps, and the average scanning speed was approximately $5 \mathrm{~mm} / \mathrm{s}$. The green dots in Fig. 5 show all the probe positions.

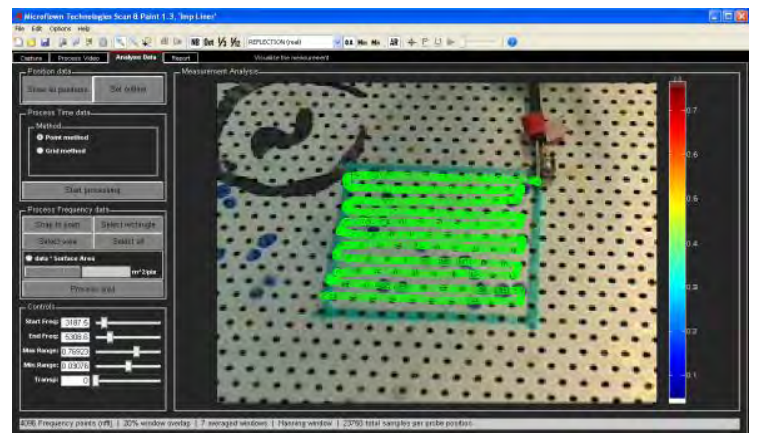

Fig. 5: Scanning measurement technique applied to absorption measurements.

The small holes of the liner are about $1 \mathrm{~mm}$ diameter, not all holes are opened. Some of them are closed with glue and they are indicated by the blue dots in the left picture (Fig.6). In the right image the colormap of reflection (real part) is plotted. The 
measurement with the PU match shows the degree of spatial resolution achievable by the method, which is in the order of a millimeter. It can be seen that all closed holes are identified with the acoustic measurement, which results in a local increase of the reflection coefficient. This test demonstrates that this absorption mapping technique is suitable for detecting defects; a procedure that may be implemented during or after the production of such liners.
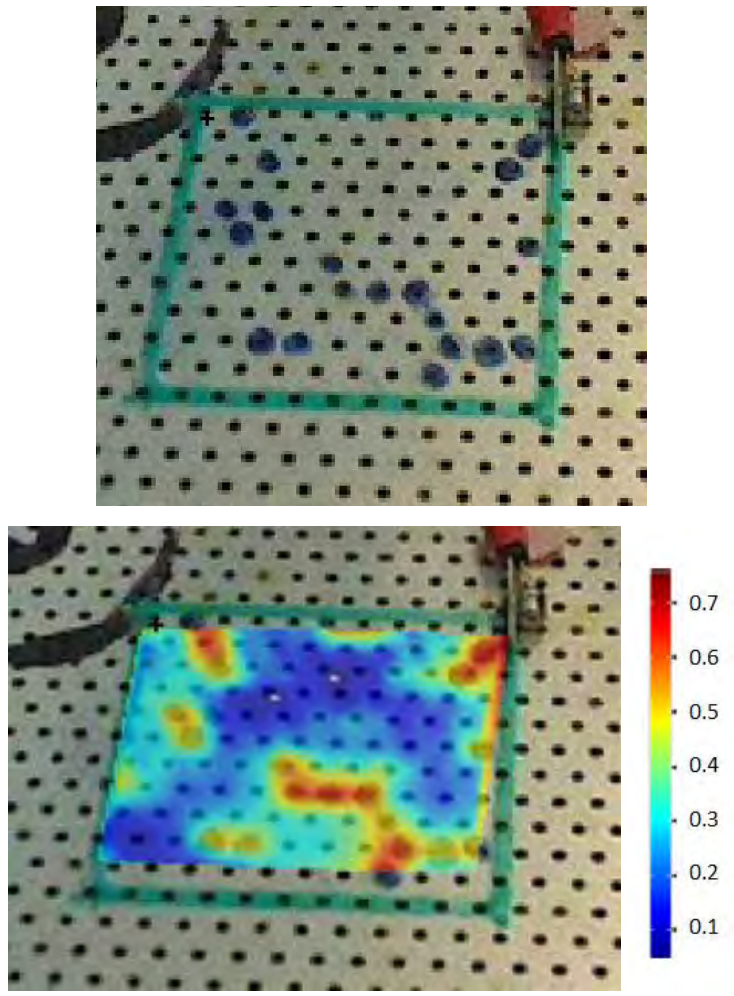

Fig. 6: Top: Liner with closed holes identified with a blue marker. Bottom: reflection coefficient colormap.

\section{High sound pressure level measurement}

For many aerospace applications it is required to test the acoustic absorption of materials under high $\mathrm{dB}$ pressure level, because such a situation resemble the real operational environment where the material must work. For this reason, a upgraded version of the standard impedance measurement set-up has been developed with which high sound levels can be generated. To cope with the elevated sound levels, a high sound pressure microphone and a high particle velocity sensor have been used. The special particle velocity sensor comprises a micro-machine perforated cap which reduces the particle velocity measured by the sensor, see Fig. 7 right.

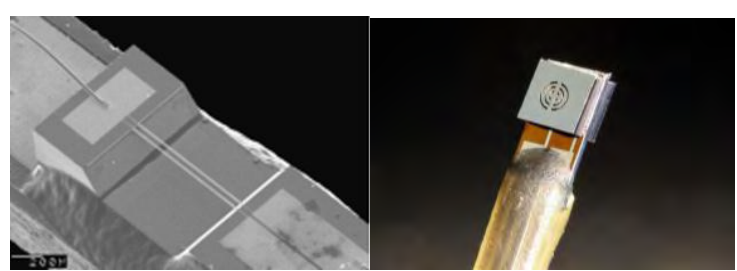

Fig. 7: Left: Regular particle velocity sensor. Right: High $d B$ particle velocity sensor [6].

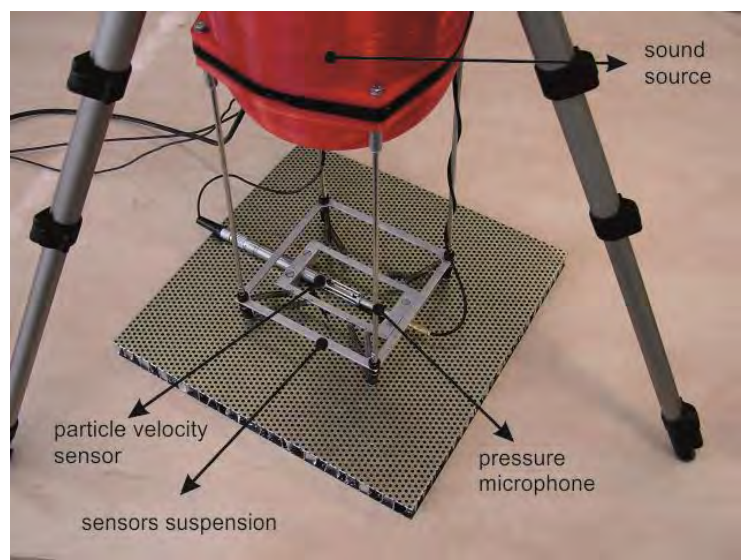

Fig. 8: System to measure the acoustic impedance at high sound pressure levels.

The set-up that has been build is shown in Fig. 8. The particle velocity sensor and microphone are positioned at a fixed distance, $27 \mathrm{~cm}$ from the sound source. The sensors are decoupled from the steel mounting by springs in order to isolate vibrations from the loudspeaker.

The sound pressure level measured at the surface is about $150 \mathrm{~dB}$ overall. In comparison to the standard in situ impedance technique, lower frequencies of around $100 \mathrm{~Hz}$ can be accessed with this set-up, mainly due to the higher pressure emission from the loudspeaker.

A measurement is performed with this measurement system, which is placed directly above a liner sample. Both sensors were positioned approx. $1 \mathrm{~cm}$ from the sample surface. The absorption results obtained by the high sound pressure level method were compared to the ones obtained with the standard impedance measurement setup and can be seen in the Fig. 9 below.

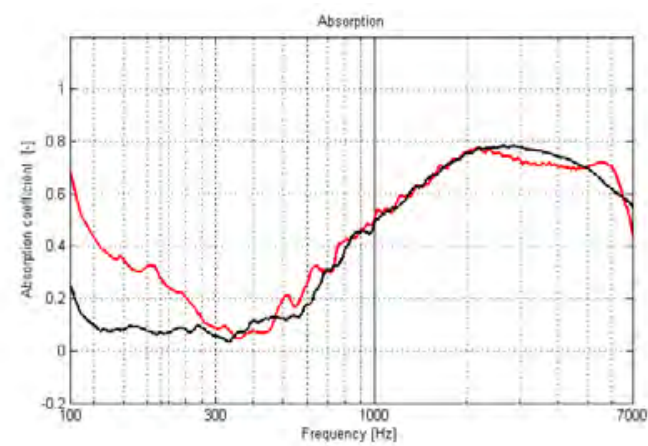


Fig. 9: Comparison between standard impedance measurement (red line) and the high sound pressure level impedance measurement (black line).

The results from the standard set-up and the high sound pressure level set-up are in good agreement for frequencies higher than $300 \mathrm{~Hz}$. At low frequencies deviations were found with the standard set-up because the sound level was insufficient.

\section{Conclusions}

In this paper the principle of measuring the absorption properties of jet engine liners with $\mathrm{PU}$ probes is investigated. Different measurements techniques have been shown as alternative to standardized methods.

Firstly, several test with jet engine liners revealed shortcomings of the open Kundt's method that is frequently used. Measurements have been performed on a typical liner with interconnected honeycomb cells. Unrealistic high absorption values were found at low frequencies because of sound leakage, and in the middle frequency range because of resonances inside the sample caused by the flange of the tube. The tests shown that for such samples more accurate results can be obtained with in situ measurements.

Secondly, with a scanning measurement technique called Scan \& Paint the local acoustic absorption properties of a liner were measured. It is shown that this technique has a very high spatial resolution, in the order of a millimetre or less, and that very small defects can be detected.

Finally, a new portable impedance set-up has been presented with which the surface impedance, reflection and absorption of a sample can be measured at sound pressure levels of up to $150 \mathrm{~dB}$ OASPL. The measurement can be performed quickly and the obtained results are similar to those obtained with the standard PU method. Although, further evaluation of the technique is required, these preliminary tests show that the method has a wide frequency range and dynamic range..

The results of the tests as shown in this paper reveal a great potential of in situ measurements of the absorption properties with combined sound pressure - particle velocity sensors. In the future, other applications may be investigated (further), such as measuring at real operating conditions where there are high sound levels, high temperatures, and high airflows.

\section{References}

[1] E. Tijs, H-E de Bree, "Recent developments free field PU impedance technique", In proceedings of SAPEM 2008

[2] H.E.de Bree, M. Nosko, E. Tijs, "A handheld device to measure the acoustic absorption in situ", In proceedings of SNVH 2008, Graz

[3] R. Lanoye, G. Vermeir, W. Lauriks, R.Kruse, V.Mellert, "Measuring the free field acoustic impedance and absorption coefficient of sound absorbing materials with a combined particle velocity sensor", JASA 119, 2826 - 2831 (2006), DOI: $10.1121 / 1.2188821$

[4] Jorge Daniel Alvarez B. and Finn Jacobsen, "An Iterative Method for Determining the Surface Impedance of Acoustic Materials In Situ", Internoise 2008, Shanghai, China

[5] Hans Elias De Bree, Microflown E-Book, chapter 6, Impedance

[6] Hans Elias De Bree, Microflown E-Book, chapter 14, High velocity

[7] E.Tijs, H-E. De Bree, P. Ferrante and A. Scofano (2008) "PU surface impedance measurements on curved liner materials in the presence of a grazing flow", 12th CEAS workshop, Bilbao, Spain. 